\title{
Consensus of Third-Order Multiagent Systems with Time Delay in Undirected Networks
}

\author{
Yanfen Cao and Yuangong Sun \\ School of Mathematical Sciences, University of Jinan, Jinan, Shandong 250022, China \\ Correspondence should be addressed to Yuangong Sun; sunyuangong@163.com
}

Received 31 March 2016; Accepted 19 May 2016

Academic Editor: Guangming Xie

Copyright (c) 2016 Y. Cao and Y. Sun. This is an open access article distributed under the Creative Commons Attribution License, which permits unrestricted use, distribution, and reproduction in any medium, provided the original work is properly cited.

\begin{abstract}
We consider consensus of a class of third-order continuous-time multiagent systems with time delay in undirected networks. By using matrix analysis and a frequency domain approach, a necessary and sufficient condition for consensus is established. A simulation result is also given to illustrate the main theoretical result.
\end{abstract}

\section{Introduction}

In recent years, more and more people are interested in multiagent systems due to their extensive applications in real world, such as cooperative control of unmanned aerial vehicle, autonomous underwater submarine, flocking, and network control in information. Among the corresponding theories for multiagent systems, consensus problem is a critical issue which has attracted interdisciplinary researchers from the fields of control, mathematics, biology, physics, computer, robot, communication and artificial intelligence, and so forth.

Most work on consensus focuses on protocols taking the form of first-order dynamics [1-5]. Extensions of consensus protocols to second-order dynamics are reported in [611]. Just as is shown in [9], the convergence of secondorder consensus algorithms relies on not only information exchange topology but also the coupling strength between the information states in contrast to first-order consensus algorithms. There is also some recent work focusing on consensus protocols with both first-order and second-order dynamics $[12,13]$.

It is well known that the dynamic equation given by second-order consensus protocols contains the derivatives of position and velocity. In practice, the dynamics of agents may involve the derivative of accelerated speed. The derivative of accelerated speed is usually called Jerk in engineering.
Jerk is a physical quantity to describe the changing rate of acceleration. A Jerk is often required in engineering, especially in transportation design and materials, and so forth. Therefore, it is necessary and significant to design a class of third-order consensus protocols by using the information of position, velocity, and accelerated speed of agents, which is the main purpose of this paper.

Recently, some people have studied some higher-order consensus protocols $[14,15]$. In [14], the authors presented some necessary and sufficient conditions for consensus of high-order multiagent systems. Consensus of multiagent systems with linear higher-order agents was considered in [15], where the authors answered whether the group converges to consensus and what consensus value it eventually reaches. However, necessary and sufficient conditions for consensus analysis of higher-order protocols in $[14,15]$ are given based on the distribution of eigenvalues of a complex matrix, which do not clearly illustrate the relationship among the eigenvalues of the involved Laplacian matrix, coupling strengths, and time delay when the system achieves consensus. On the other hand, higher-order consensus protocols with time delay receive less attention in the literature. Therefore, in this paper we will introduce a third-order consensus protocol with time delay and provide a convergence analysis for the given third-order consensus protocol in undirected networks. A necessary and sufficient condition for consensus is established, which clearly shows the relationship among 
the nonzero eigenvalues of the Laplacian matrix, coupling strengths, and time delay when the system achieves consensus asymptotically.

An outline of this paper is as follows. In Section 2, some preliminaries about graph and a class of third-order protocol are introduced. The main result of this paper is proposed in Section 3. In Section 4, a simulation result is given to illustrate the main result. Section 5 concludes the whole paper.

Notations. The following notations will be used throughout this paper. $\|\cdot\|$ denotes Euclidean norm. $\mathbf{j}$ is the imaginary unit. $\otimes$ denotes the Kronecker product. $R^{n}$ and $R^{N \times N}$ denote the real space of $n$-dimensional column vectors and $N \times$ $N$-dimensional matrices, respectively. $I_{N}\left(O_{N}\right) \in R^{N \times N}$ is an $N \times N$-dimensional identity (zero) matrix and $\mathbf{1}_{\mathbf{N}}=$ $(\mathbf{1}, \ldots, \mathbf{1})^{\mathrm{T}}\left(\mathbf{0}_{\mathrm{N}}=(\mathbf{0}, \ldots, \mathbf{0})^{\mathrm{T}}\right)$ denotes the $N$-dimensional column vector with entries $1(0)$.

\section{Preliminaries}

2.1. Graph Theory. Let $G=(\nu, \mathcal{\varepsilon}, A)$ be an $N$-order weighted undirected graph with set of nodes $v=\left\{\vartheta_{1}, \vartheta_{2}, \ldots, \vartheta_{N}\right\}$, set of edges $\varepsilon=\left\{\left(\vartheta_{i}, \vartheta_{j}\right): \vartheta_{i}, \vartheta_{j} \in \nu\right\} \subseteq \nu \times \nu$, and weighted adjacent matrix $A=\left(a_{i j}\right)_{N \times N}$. An edge of $G$ is denoted by $e_{i j}=\left(\vartheta_{i}, \vartheta_{j}\right)$. In the undirected graph, $e_{i j} \in \varepsilon \Leftrightarrow e_{j i} \in \varepsilon$. The adjacency matrix is defined as $a_{i i}=0$ and $a_{i j}=a_{j i} \geq 0$ for $i \neq j$ and $i, j \in\{1,2, \ldots, N\}$, where $a_{i j}>0 \Leftrightarrow e_{i j} \in \varepsilon$. A path is a sequence of edges of the form $\left(\vartheta_{i 1}, \vartheta_{i 2}\right),\left(\vartheta_{i 2}, \vartheta_{i 3}\right), \ldots$, where $\vartheta_{i j} \in \nu$. If there is a path for arbitrary two nodes in the graph, we call that the graph is connected.

The Laplacian matrix of the graph $G$ is defined as $L=$ $\left[l_{i j}\right] \in R^{N \times N}$, where

$$
l_{i i}=-\sum_{j=1, j \neq i}^{N} l_{i j}, \quad l_{i j}=-a_{i j}, \quad i \neq j .
$$

Obviously, the Laplacian matrix $L$ is a positive semidefinite matrix and satisfies $l_{i i} \geq 0, l_{i j} \leq 0$ for $i \neq j$ and $\sum_{j=1}^{N} l_{i j}=0$ for $i=1, \ldots, N$.

The following lemma is well known.

Lemma 1 (see [2]). If the undirected graph $G$ is connected, its Laplacian $L$ has a simple zero eigenvalue with the associated eigenvector $\mathbf{1}_{N}$ and all the other eigenvalues are positive real numbers.

2.2. Third-Order Consensus Protocol. Suppose the dynamics of each node is described by the following third-order system:

$$
\begin{aligned}
\dot{x}_{i}(t) & =v_{i}(t), \\
\dot{v}_{i}(t) & =z_{i}(t), \\
\dot{z}_{i}(t) & =u_{i}(t), \\
& i=1,2, \ldots, N,
\end{aligned}
$$

where $t \geq 0, x_{i}(t) \in R^{n}, v_{i}(t) \in R^{n}$, and $z_{i}(t) \in R^{n}$ represent the position, velocity, and accelerated speed of node $\vartheta_{i}$, respectively, and $u_{i}(t) \in R^{n}$ is the control input (or protocol) to be designed.

In this paper we consider the following third-order protocol:

$$
\begin{aligned}
u_{i}(t)= & k_{1} \sum_{j=1}^{N} a_{i j}\left(x_{j}(t-\tau)-x_{i}(t-\tau)\right) \\
& +k_{2} \sum_{j=1}^{N} a_{i j}\left(v_{j}(t-\tau)-v_{i}(t-\tau)\right) \\
& +k_{3} \sum_{j=1}^{N} a_{i j}\left(z_{j}(t-\tau)-z_{i}(t-\tau)\right),
\end{aligned}
$$

where $i=1,2, \ldots, N, k_{1}>0, k_{2}>0$, and $k_{3}>0$ are appropriate coupling strengths.

Definition 2. Say system (2) with protocol (3) achieves consensus asymptotically, if $\lim _{t \rightarrow \infty}\left\|x_{i}(t)-x_{j}(t)\right\|=0$, $\lim _{t \rightarrow \infty}\left\|v_{i}(t)-v_{j}(t)\right\|=0$, and $\lim _{t \rightarrow \infty}\left\|z_{i}(t)-z_{j}(t)\right\|=0$ for any $i, j=1,2, \ldots, N$ and any initial condition $x(t)=\phi(t)$, where $\phi$ is a continuous function defined on $[-\tau, 0]$.

We first have the following lemma for system (2) with protocol (3).

Lemma 3. If system (2) with protocol (3) achieves consensus asymptotically, one has

$$
\begin{aligned}
& \lim _{t \rightarrow \infty}\left\|x_{i}(t)-\frac{1}{N} \sum_{j=1}^{N}\left[x_{j}(0)+v_{j}(0) t+\frac{1}{2} z_{j}(0) t^{2}\right]\right\| \\
& \quad=0 \\
& \lim _{t \rightarrow \infty}\left\|v_{i}(t)-\frac{1}{N} \sum_{j=1}^{N}\left[v_{j}(0)+z_{j}(0) t\right]\right\|=0, \\
& \lim _{t \rightarrow \infty}\left\|z_{i}(t)-\frac{1}{N} \sum_{j=1}^{N} z_{j}(0)\right\|=0 .
\end{aligned}
$$

Proof. (i) Since the graph is undirected, by (2) and (3), we have $\sum_{j=1}^{N} \dot{z}_{j}(t)=\mathbf{0}$. Integrating it on both sides from 0 to $t$, we have

$$
\sum_{j=1}^{N} z_{j}(t)=\sum_{j=1}^{N} z_{j}(0) .
$$

Denote $e_{i j}(t)=z_{j}(t)-z_{i}(t)(i, j=1, \ldots, N, j \neq i)$. Thus, (5) will be rewritten into the following form:

$$
N z_{i}(t)+\sum_{j=1, j \neq i}^{N} e_{i j}(t)=\sum_{j=1}^{N} z_{j}(0) .
$$

Namely,

$$
z_{i}(t)-\frac{1}{N} \sum_{j=1}^{N} z_{j}(0)=-\frac{1}{N} \sum_{j=1, j \neq i}^{N} e_{i j}(t) .
$$


Note that the system achieves consensus; we have $\lim _{t \rightarrow \infty}\left\|e_{i j}(t)\right\|=0$ for $i, j=1, \ldots, N, j \neq i$. Therefore, we get from (7) that

$$
\lim _{t \rightarrow \infty}\left\|z_{i}(t)-\frac{1}{N} \sum_{j=1}^{N} z_{j}(0)\right\|=0 .
$$

(ii) By (2) and (5), we have

$$
\sum_{j=1}^{N} \dot{v}_{j}(t)=\sum_{j=1}^{N} z_{j}(t)=\sum_{j=1}^{N} z_{j}(0) .
$$

Integrating the above equality from 0 to $t$ yields

$$
\sum_{j=1}^{N} v_{j}(t)=\sum_{j=1}^{N}\left(v_{j}(0)+z_{j}(0) t\right)
$$

Similar to the discussion in (i), we can easily get the second equality of (4).

(iii) By (2) and (10), we have

$$
\sum_{j=1}^{N} \dot{x}_{j}(t)=\sum_{j=1}^{N} v_{j}(t)=\sum_{j=1}^{N}\left(v_{j}(0)+z_{j}(0) t\right) .
$$

By integrating it from 0 to $t$, we get

$$
\sum_{j=1}^{N} x_{j}(t)=\sum_{j=1}^{N}\left(x_{j}(0)+v_{j}(0) t+\frac{1}{2} z_{j}(0) t^{2}\right) .
$$

We can similarly derive the first equality of (4) as above. The proof of Lemma 3 is complete.

\section{Main Result}

In the sequel, set

$$
\begin{aligned}
& \xi(t) \\
& \quad=\left(x_{1}^{T}(t), v_{1}^{T}(t), z_{1}^{T}(t), \ldots, x_{N}^{T}(t), v_{N}^{T}(t), z_{N}^{T}(t)\right)^{T} .
\end{aligned}
$$

System (2) with protocol (3) can be rewritten into

$$
\dot{\xi}(t)=\left(I_{N} \otimes A\right) \xi(t)-(L \otimes B) \xi(t-\tau),
$$

where

$$
\begin{aligned}
A & =\left(\begin{array}{lll}
0 & 1 & 0 \\
0 & 0 & 1 \\
0 & 0 & 0
\end{array}\right) \otimes I_{n}, \\
B & =\left(\begin{array}{lll}
0 & 0 & 0 \\
0 & 0 & 0 \\
k_{1} & k_{2} & k_{3}
\end{array}\right) \otimes I_{n} .
\end{aligned}
$$

By Lemma 3, let

$$
\bar{\delta}(t)=\xi(t)-\mathbf{1}_{\mathbf{N}} \otimes\left(\alpha^{\mathrm{T}}(\mathbf{t}), \beta^{\mathrm{T}}(\mathbf{t}), \gamma^{\mathrm{T}}\right)^{\mathrm{T}},
$$

where

$$
\begin{aligned}
\alpha(t) & =\frac{1}{N} \sum_{j=1}^{N}\left(x_{j}(0)+v_{j}(0) t+\frac{z_{j}(0) t^{2}}{2}\right), \\
\beta(t) & =\frac{1}{N} \sum_{J=1}^{N}\left(v_{j}(0)+z_{j}(0) t\right), \\
\gamma & =\frac{1}{N} \sum_{j=1}^{N} z_{j}(0) .
\end{aligned}
$$

A straightforward computation yields that system (14) is equivalent to

$$
\dot{\bar{\delta}}=\left(I_{N} \otimes A\right) \bar{\delta}(t)-(L \otimes B) \bar{\delta}(t-\tau) .
$$

By Lemma 1, we can denote the nonzero eigenvalues of $L$ by $\lambda_{2}, \lambda_{3}, \ldots, \lambda_{N}$ and set $0<\lambda_{2} \leq \lambda_{3} \leq \cdots \leq \lambda_{N}$. Then there exists an orthogonal matrix $W$ such that

$$
\begin{aligned}
\bar{\Lambda} & =W^{T} L W=\operatorname{diag}\left\{0, \lambda_{2}, \ldots, \lambda_{N}\right\}, \\
\left(W^{T} \otimes I_{3}\right) \bar{\delta}(t) & =\left[\mathbf{0}_{n}^{T}, \mathbf{0}_{n}^{T}, \mathbf{0}_{n}^{T}, \delta^{T}(t)\right]^{T},
\end{aligned}
$$

where $\delta(t)$ is a column vector of appropriate dimension. Premultiplying both sides of (18) by $W^{T} \otimes I_{3}$, we have

$$
\dot{\delta}(t)=\left(I_{N-1} \otimes A\right) \delta(t)-(\Lambda \otimes B) \delta(t-\tau),
$$

where $\Lambda=\operatorname{diag}\left\{\lambda_{2}, \ldots, \lambda_{N}\right\}$.

Theorem 4. System (2) with protocol (3) achieves consensus asymptotically if and only if

$$
\frac{\lambda_{i}\left(k_{2} \omega \cos (\omega \tau)+k_{3} \omega^{2} \sin (\omega \tau)-k_{1} \sin (\omega \tau)\right)}{\omega^{3}}<1,
$$

where $i=2,3, \ldots, N ; \omega$ satisfies the following equation:

$$
k_{2} \omega \tan (\omega \tau)=k_{3} \omega^{2}-k_{1} \text {. }
$$

Proof. Based on the above analysis, consensus of system (14) is equivalent to the asymptotic stability of system (20). On the other hand, system (20) is asymptotically stable if and only all its eigenvalues lie on the open left half plane (LHP). Thus, it only requires showing that all the eigenvalues of system (20) lie on the open LHP if and only if (21) and (22) hold. By using Laplace transform on both sides of (20), we have $\delta(s)=G_{\tau}^{-1}(s) \delta(0)$, where

$$
G_{\tau}(s)=s I_{3 n(N-1)}-I_{N-1} \otimes A+(\Lambda \otimes B) e^{-\tau s} .
$$

Note that

$$
\begin{aligned}
I_{N-1} & \otimes A-(\Lambda \otimes B) e^{\tau s} \\
\quad & =\operatorname{diag}\left\{A-\lambda_{2} e^{\tau s} B, \ldots, A-\lambda_{n} e^{\tau s} B\right\} .
\end{aligned}
$$


By a direct calculation, we get the determinant of $G_{\tau}(s)$ :

$$
\begin{aligned}
& \operatorname{det}\left(G_{\tau}(s)\right)=\prod_{i=2}^{N} \operatorname{det}\left(s I_{3 n}-A+\lambda_{i} e^{-\tau s} B\right) \\
& =\prod_{i=2}^{N} \operatorname{det}\left(\left(s I_{3}-M_{i}\right) \otimes I_{n}\right) \\
& =\prod_{i=2}^{N}\left(s^{3}+\lambda_{i} k_{3} e^{-\tau s} s^{2}+\lambda_{i} k_{2} e^{-\tau s} s+\lambda_{i} k_{1} e^{-\tau s}\right)^{n},
\end{aligned}
$$

where

$$
M_{i}=\left(\begin{array}{ccc}
0 & 1 & 0 \\
0 & 0 & 1 \\
-\lambda_{i} k_{1} & -\lambda_{i} k_{2} & -\lambda_{i} k_{3}
\end{array}\right), \quad i=2,3, \ldots, N .
$$

By (25), all the zeros of $G_{\tau}(s)$ lie on the open LHP if and only if all the roots of equations

$$
\begin{aligned}
s^{3}+\lambda_{i} k_{3} e^{-\tau s} s^{2}+\lambda_{i} k_{2} e^{-\tau s} s+\lambda_{i} k_{1} e^{-\tau s}= & 0, \\
& i=2, \ldots, N,
\end{aligned}
$$

are all located on the open LHP. Since (27) is equivalent to

$$
1+\frac{\lambda_{i} k_{3} e^{-\tau s} s^{2}+\lambda_{i} k_{2} e^{-\tau s} s+\lambda_{i} k_{1} e^{-\tau s}}{s^{3}}=0,
$$

for $i=2, \ldots, N$, all the roots of (27) lie on the open LHP if and only if all the roots of (28) are located on the open LHP. By the Nyquist stability criterion, all the roots of (28) lie on the open LHP if and only if the Nyquist curve

$$
z=\frac{\lambda_{i} k_{3} e^{-\tau \mathbf{j} \omega}(\mathbf{j} \omega)^{2}+\lambda_{i} k_{2} e^{-\tau \mathbf{j} \omega} \mathbf{j} \omega+\lambda_{i} k_{1} e^{-\tau \mathbf{j} \omega}}{(\mathbf{j} \omega)^{3}}
$$

does not enclose the point $(-1,0 \mathbf{j})$, where $\mathbf{j}$ is an imaginary number unit and $i=2, \ldots, N$. Using Euler's formula for (29), we have

$$
\begin{aligned}
z= & \frac{-\lambda_{i}\left(k_{2} \omega \cos (\omega \tau)+k_{3} \omega^{2} \sin (\omega \tau)-k_{1} \sin (\omega \tau)\right)}{\omega^{3}} \\
+ & \frac{\left(-k_{3} \omega^{2} \cos (\omega \tau)+k_{2} \omega \sin (\omega)+k_{1} \cos (\omega \tau)\right) \lambda_{i}}{\omega^{3}}
\end{aligned}
$$

$$
\text { j. }
$$

Hence, all the Nyquist curves (29) do not enclose the point $(-1,0 \mathbf{j})$ if and only if $(21)$ holds, where $\omega$ is the solution of equation

$$
\left(-k_{3} \omega^{2} \cos (\omega \tau)+k_{2} \omega \sin (\omega)+k_{1} \cos (\omega \tau)\right) \lambda_{i}=0 .
$$

Namely, (22) holds. The proof of Theorem 4 is complete.

For the particular case when $\tau=0$, Theorem 4 reduces to Corollary 5 in [16].

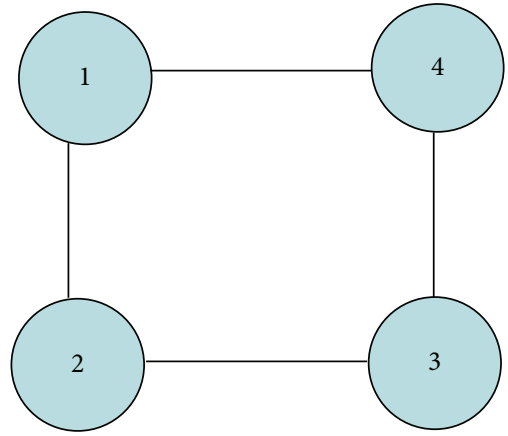

FIgURE 1: An undirected graph.

Corollary 5. System (2) with protocol (3) and $\tau=0$ achieves consensus asymptotically if and only if the coupling strengths $k_{1}, k_{2}$, and $k_{3}$ and the eigenvalue $\lambda_{2}$ of the Laplacian matrix $L$ satisfy

$$
\lambda_{2}>\frac{k_{1}}{k_{2} k_{3}}
$$

\section{Simulation}

In this section, we will give a simulation to illustrate the main result. The topology graph in the simulation is shown in Figure 1 which has four agents and 0-1 weights.

It is easy to see the corresponding Laplacian matrix

$$
L=\left[\begin{array}{cccc}
2 & -1 & 0 & -1 \\
-1 & 2 & -1 & 0 \\
0 & -1 & 2 & -1 \\
-1 & 0 & -1 & 2
\end{array}\right]
$$

If we consider $k_{1}=k_{2}=k_{3}=1$ and $\tau=0.3$, conditions (21) and (22) are satisfied. By Theorem 4, consensus of system (2) with protocol (3) is achieved asymptotically. However, when $\tau=0.356$, conditions (21) and (22) are not satisfied. Therefore, system (2) with protocol (3) does not achieve consensus asymptotically. The states of position, velocity, and accelerated speed of agents with $\tau=0.3$ and $\tau=0.356$ are shown in Figures 2 and 3, respectively.

\section{Conclusions}

In this paper, we study the consensus problem of the third-order dynamic multiagent system with time delay in undirected graphs. A necessary and sufficient condition for consensus of the system has been established. A simulation result illustrates the effectiveness of the theoretical result. Consensus of third-order multiagent systems with time delay in directed graphs will be further studied in the future.

\section{Competing Interests}

The authors declare that they have no competing interests. 


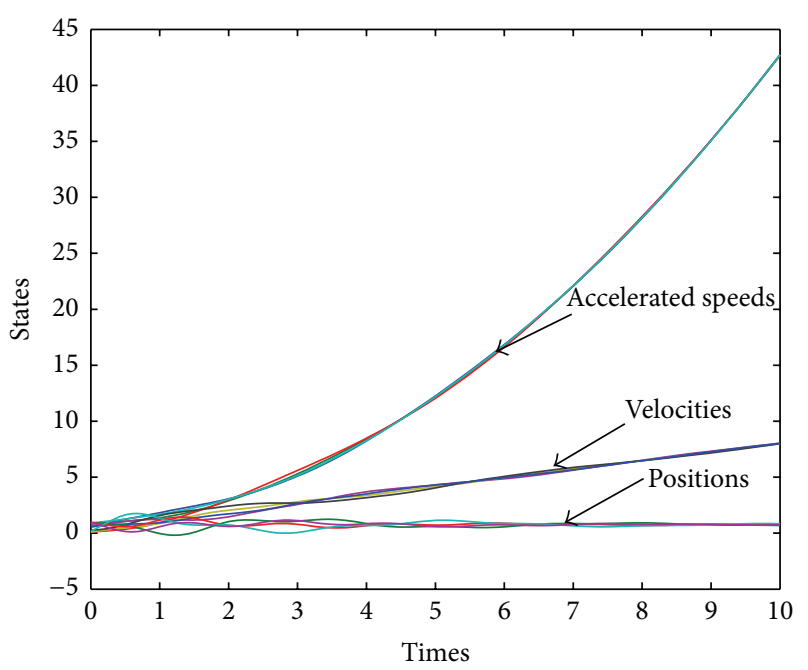

Figure 2: Trajectories of system (2) with $k_{1}=k_{2}=k_{3}=1, \tau=0.3$.

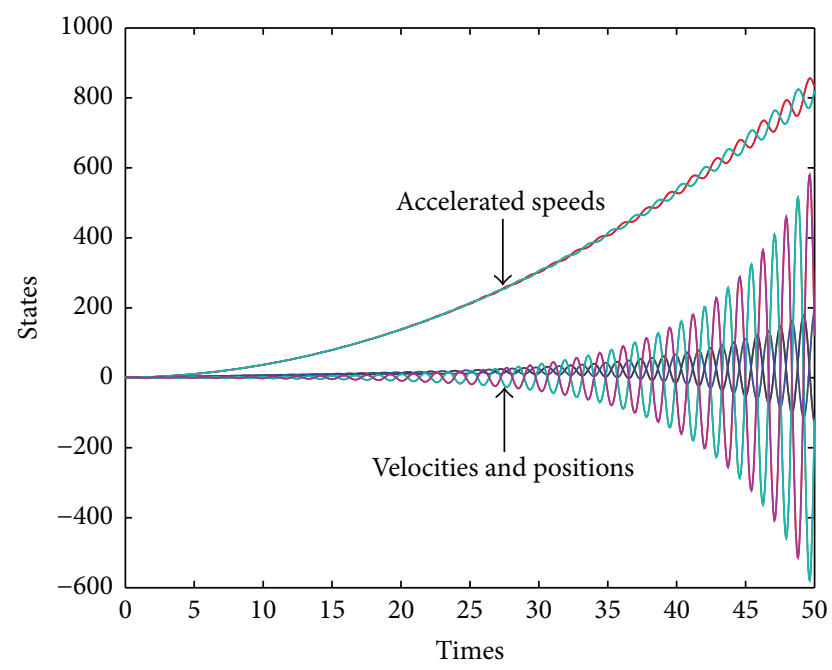

Figure 3: Trajectories of system (2) with $k_{1}=k_{2}=k_{3}=1, \tau=$ 0.356 .

\section{Acknowledgments}

This work was supported by the Natural Science Foundation of Shandong Province under Grant no. JQ201119 and the National Natural Science Foundation of China under Grant nos. 61174217, 61374074, and 61473133 .

\section{References}

[1] A. Jadbabaie, J. Lin, and A. S. Morse, "Coordination of groups of mobile autonomous agents using nearest neighbor rules," IEEE Transactions on Automatic Control, vol. 48, no. 6, pp. 988-1001, 2003.

[2] R. Olfati-Saber and R. M. Murray, "Consensus problems in networks of agents with switching topology and time-delays," IEEE Transactions on Automatic Control, vol. 49, no. 9, pp. 15201533, 2004.

[3] W. Ren and R. W. Beard, "Consensus seeking in multiagent systems under dynamically changing interaction topologies,"
IEEE Transactions on Automatic Control, vol. 50, no. 5, pp. 655661, 2005.

[4] J. Shen and J. Cao, "Consensus of multi-agent systems on time scales," IMA Journal of Mathematical Control and Information, vol. 29, no. 4, pp. 507-517, 2012.

[5] Z. Ji, H. Lin, and H. Yu, "Protocols design and uncontrollable topologies construction for multi-agent networks," IEEE Transactions on Automatic Control, vol. 60, no. 3, pp. 781-786, 2015.

[6] W. Ren and R. W. Beard, Distributed Consensus in Multi-Vehicle Cooperative Control, Spring, London, UK, 2008.

[7] W. Yu, G. Chen, M. Cao, and J. Kurths, "Second-Order consensus for multiagent systems with directed topologies and nonlinear dynamics," IEEE Transactions on Systems, Man, and Cybernetics, Part B: Cybernetics, vol. 40, no. 3, pp. 881-891, 2010.

[8] W. Yu, G. Chen, and M. Cao, "Some necessary and sufficient conditions for second-order consensus in multi-agent dynamical systems," Automatica, vol. 46, no. 6, pp. 1089-1095, 2010.

[9] W. Ren, "Second-order consensus algorithm with extensions to switching topologies and reference models," in Proceedings of the American Control Conference (ACC '07), pp. 1431-1436, July 2007.

[10] G.-H. Xu, Z.-H. Guan, D.-X. He, M. Chi, and Y.-H. Wu, "Distributed tracking control of second-order multi-agent systems with sampled data," Journal of the Franklin Institute, vol. 351, no. 10, pp. 4786-4801, 2014.

[11] H. Pei, S. Chen, and Q. Lai, "A local flocking algorithm of multiagent dynamic systems," International Journal of Control, vol. 88, no. 11, pp. 2242-2249, 2015.

[12] Y. Zheng, Y. Zhu, and L. Wang, "Consensus of heterogeneous multi-agent systems," IET Control Theory and Applications, vol. 5, no. 16, pp. 1881-1888, 2011.

[13] Y. Zheng and L. Wang, "Finite-time consensus of heterogeneous multi-agent systems with and without velocity measurements," Systems \& Control Letters, vol. 61, no. 8, pp. 871-878, 2012.

[14] W. Ren, K. Moore, and Y. Q. Chen, "High-order consensus algorithms in cooperative vehicle systems," in Proceedings of the IEEE International Conference on Networking, Sensing and Control, pp. 457-462, Fort Lauderdale, Fla, USA, 2006.

[15] P. Wieland, J. S. Kim, H. Scheu, and F. Allgöwer, "On consensus in multi-agent systems with linear high-order agents," in Proceedings of the 17th World Congress of the International Federation of Automatic Control, pp. 1541-1546, Seoul, Republic of Korea, July 2008.

[16] Y. Cao and Y. Sun, "Consensus analysis for third-order multiagent systems in directed networks," Mathematical Problems in Engineering, vol. 2015, Article ID 940823, 9 pages, 2015. 


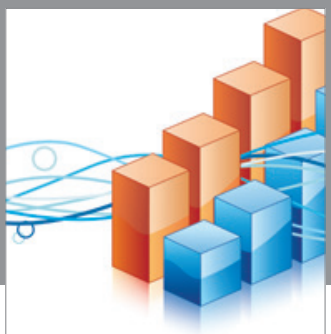

Advances in

Operations Research

vatem alat4

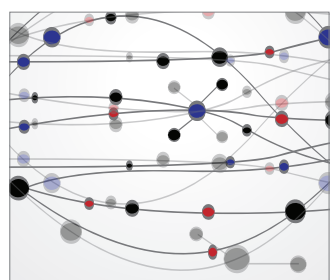

\section{The Scientific} World Journal
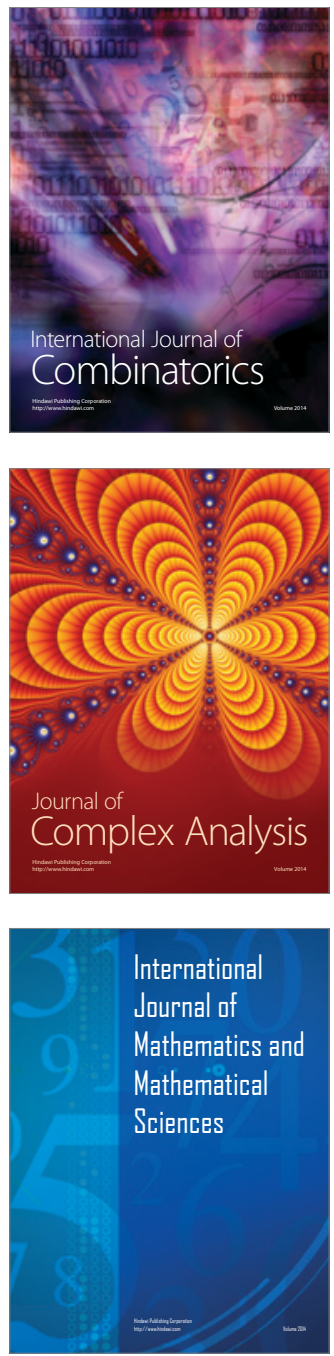
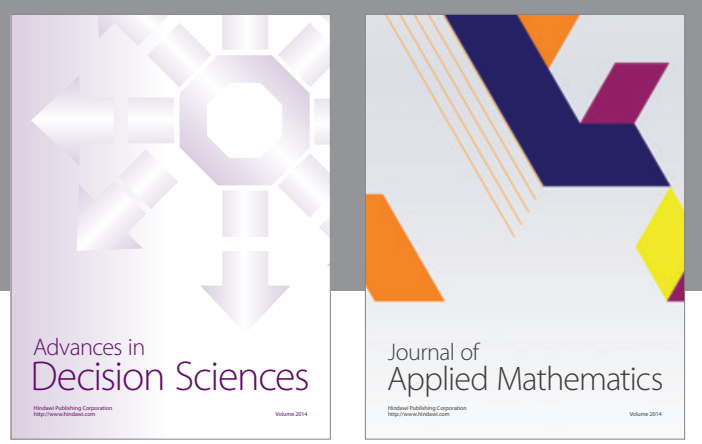

Algebra

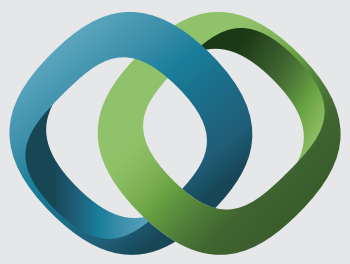

\section{Hindawi}

Submit your manuscripts at

http://www.hindawi.com
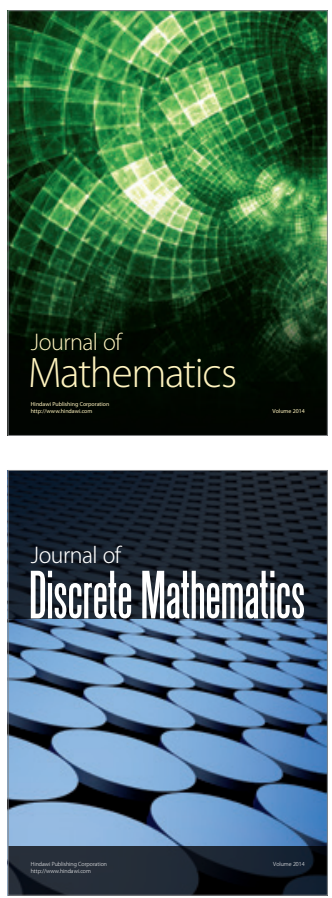

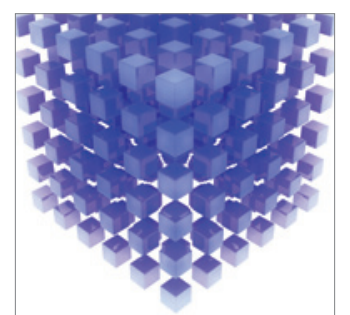

Mathematical Problems in Engineering
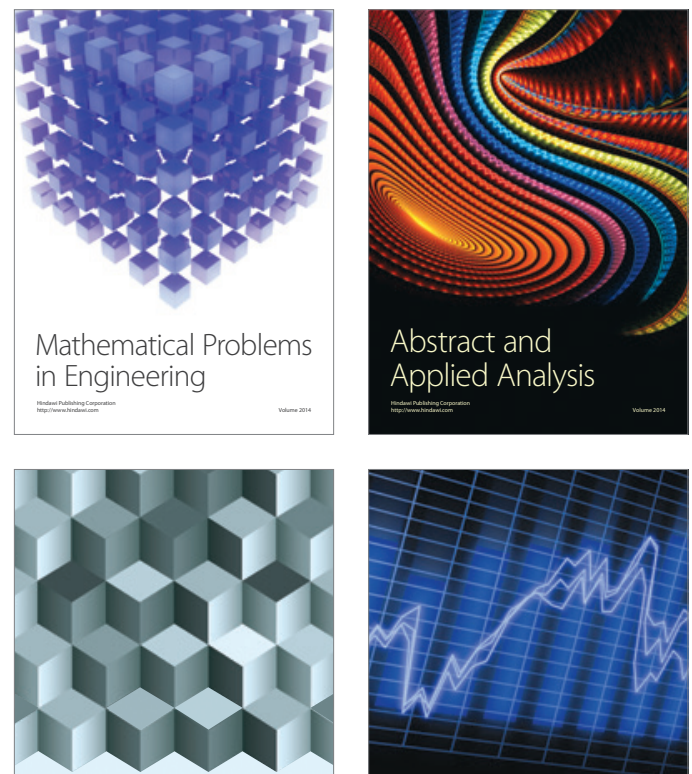

Journal of

Function Spaces

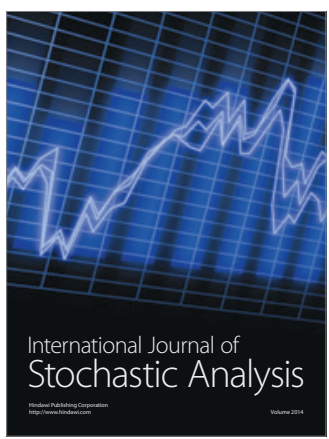

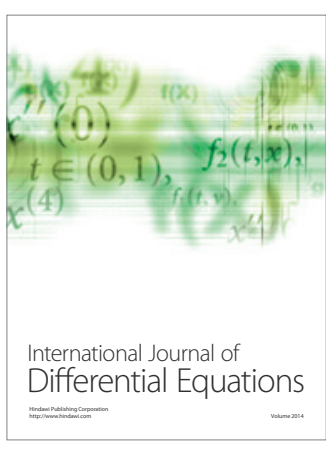
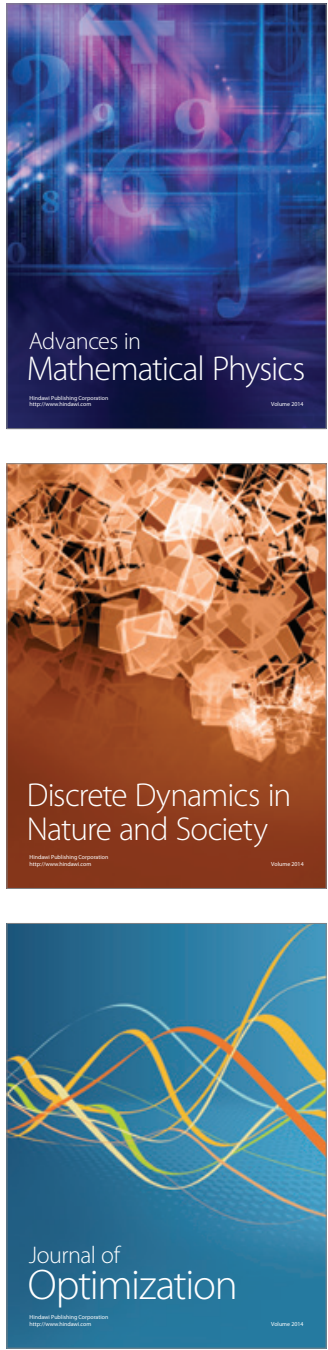